\title{
Surface texture characterization of alpha-beta titanium alloy (Ti6Al4V) after hydrostatic burnishing
}

\author{
$S$ Swirad* $^{*}$ \\ Rzeszow University of Technology, The Faculty of Mechanical Engineering and Aeronautics, al. \\ Powstancow Warszawy 12, 35-959 Rzeszow, Poland
}

\begin{abstract}
The ball burnishing process with hydrostatic tools is very economical finishing process for various types of machine parts. This process reduces the height of surface unevenness, introduces compressive stresses at high depth (approx. $1 \mathrm{~mm}$ ) and increase the hardness of the surface layer. The paper presents effect of the ball burnishing process parameters with hydrostatic tools on the surface structure geometry of titanium alloy. This shows how the surface roughness can be reduced after preceding operation by the ball burnishing process. This process changes the surface stereometrics of the previously milled surface. Burnishing with hydrostatic tools can be easily and effectively used either on conventional or on $\mathrm{CNC}$ machines. The Ecoroll burnishing system used in this research consists of a high-pressure hydraulic pump (model 4.0 HGP) and the $6 \mathrm{~mm}$ diameter burnishing tool both connected via high-pressure hoses. The burnishing tool is based on a hydrostatics principle; the key element is a $6 \mathrm{~mm}$ ball made of ceramics. The input process parameters considered in the paper included: burnishing rate, applied pressure and line to line pitch. It also showed a positive effect of hydrostatics burnishing on roughness and geometric structure of the surface. In most cases, the result is anisotropic surface, reduced roughness, reduced amplitude values of parameters such as: Sa, Sz. The biggest impact on the value of indicators of geometric structure comes from pressure, the smaller (but still significant) impact results from a tool speed and line to line pitch
\end{abstract}

\section{Introduction}

Materials such as titanium and its alloys, in special Ti6Al4V, are widely used in military, aircraft, spacecraft and medical industry. The Ti-6Al-4V alloy is one of the most utilized Ti alloys, accounting for more than $50 \%$ of total titanium usage [1]. Low thermal conductivity and the volume specific heat of these materials result in very high cutting temperature during machining. In addition, the ability of these materials to maintain strength at high temperature and severe work hardening lead to higher machining forces [2]. Titanium alloys are generally accepted as "difficult-to-machine" materials. Furthermore, the ability to change phase of titanium alloys makes the deformation process more complex. Titanium

\footnotetext{
* Corresponding author: s.swirad@prz.edu.pl
} 
and its alloy have excellent weight ratio to strength which is maintained at high temperature and very high corrosion resistance. However, Ti-6Al-4V alloy is known to possess poor wear resistance that restricts its applications particularly in areas involving wear and friction $[3,4]$. The ball burnishing process with hydrostatic tools is very economical finishing process for various types of machine parts. Burnishing tools can be used with both conventional and numerically controlled turning and milling machines [5]. Hydrostatic burnishing tools increases the fatigue strength of metal components. Both low and high cycle fatigue resistance is increased and stress corrosion cracking is prevented or reduced [6]. This process is well suited for treating both rotationally symmetrical parts and freeform surfaces. In ball burnishing - ball is pressed against the workpiece surface, plasticizing the material's surface layer and changing the surface`s micro-structure. At the contact point, the deep rolling force generates Hertzian contact stresses in the material's rim zone. If this stress is higher than the material's yield strength, the material near the surface starts to flow. After the process, residual compressive stress remain in the zone, increasing the material fatigue strength.

Burnishing can be used widely on various materials such as aluminum, steel $[7,8]$, alloys $[9,10]$ and brass [11]. The effect of ball burnishing on surface stereometrics structure is the most commonly described in paper reviews. The most concern parameters are feed, force and speed. Force plays a vital role in the ball burnishing process. From the start of the plastics deformation, an increase in force causes surface roughness to decrease until it reaches to a reasonably low value. Due to the overly high force may results in spalling of the surface $[8,10]$.

\section{Experimental Investigation}

\subsection{Materials and experimental equipment}

Experimental investigation was carried out on flat bar of titanium alloy Ti-6Al-V. Titanium alloy Ti-6Al-V, work material is a $(\alpha+\beta)$ which was commercially available of aerospace grade 5. Chemical composition of the material is given in table 1 . The turning and burnishing tests were performed on Hass VF-1 vertical CNC machining center of $22 \mathrm{~kW}$ spindle power with a maximum spindle speed of $8000 \mathrm{rpm}$. Initially, titanium bars were milled using, cutting speed of $155 \mathrm{~m} / \mathrm{min}$, depth of cut of $0.2 \mathrm{~mm}$ and feed of $0.075 \mathrm{~mm} /$ tooth. A carbide tipped end mill insert of $12 \mathrm{~mm}$ was used as the cutting tool. The effect of the burnishing process depends on the initial surface roughness. The machined average roughness was found to be in the range $0.60 \mu \mathrm{m}$.

Table 1. Chemical composition of titanium alloy Ti-6Al-4V.

\begin{tabular}{|c|c|c|c|c|c|c|c|c|c|}
\hline \multirow{3}{*}{ Wt. \% } & \multicolumn{9}{|c|}{ Element } \\
\cline { 2 - 11 } & $\mathrm{Ti}$ & $\mathrm{Al}$ & $\mathrm{V}$ & $\mathrm{Fe}$ & $\mathrm{O}$ & $\mathrm{C}$ & $\mathrm{N}$ & $\mathrm{Y}$ & $\mathrm{H}$ \\
\cline { 2 - 11 } & Balanced & 6.1 & 4 & 0.16 & 0.11 & 0.02 & 0.01 & 0.001 & 0.001 \\
\hline
\end{tabular}

The Ecoroll burnishing system used in this research consists of a high-pressure hydraulic pump (model 4.0 HGP) and the $6 \mathrm{~mm}$ diameter burnishing tool both connected via high-pressure hoses. Pressure was supplied by the hydraulic pump, which is equipped with its own coolant tank and pump at the coolant maximum pressure of $40 \mathrm{MPa}$. The burnishing ball is placed in the tool in (replaceable) slot where it can be rotated in either direction. Additionally, the tool head can "move freely" with approx. 6-8 $\mathrm{mm}$ (without changing the pressure), which allows for eliminating small errors (e.g. the shape) of the workpiece. 
The tests were carried out according to the static, determined, sequential, polyselectional Hartley's plan, as recommended in the study of surface layer [12]. Depending on the distance and location of experimental points (in the space of operation factors) with respect to the central point (zero), these plans can be built on hypersphere or hypercube. Group of plans built on a hypercube, requires experiments only on three equally spaced levels of variability of operation factors [13]. Such plan as to obtain the mathematical model of the process tested in the form of a polynomial of the second degree was assumed in this work. It consists of three blocks of experiments - fractional repetition of experiment type PS/DK 2n, experiment in stellar points with arm $\alpha=1$ and experiment in the plan central point.

The three-level planning is done encoding the factors of input variables at three levels, higher - marked as ,"+, mean (basic, zero) - marked as „,0” and lower- marked as „,-,. The burnishing process parameters considered for this study were burnishing pressure force, burnishing speed and burnishing width. Other parameters were held constant, as their effect was not significant as seen from the preliminary studies. The design matrix is given in Table 2.

Table 2. Ball burnishing process parameters and their identified levels.

\begin{tabular}{|c|c|c|c|c|}
\hline \multirow{2}{*}{ Code } & \multirow{2}{*}{ Control factor } & \multicolumn{3}{|c|}{ Level } \\
\cline { 3 - 5 } & & 1 & 2 & 3 \\
\hline A & Pressure force $(\mathrm{P}),[\mathrm{MPa}]$ & 10 & 15 & 20 \\
\hline B & Speed $(\mathrm{v}),[\mathrm{mm} / \mathrm{min}]$ & 1000 & 2000 & 3000 \\
\hline C & Width $(\mathrm{a}),[\mathrm{mm}]$ & 0.4 & 0.6 & 0.8 \\
\hline
\end{tabular}

\section{Results and discussion}

The sample was measured by Talysurf CCI white light interferometer. The number of measurement points was $1024 \times 1024$ in the area of $3.3 \times 3.3 \mathrm{~mm}$., height resolution was $0.01 \mathrm{~nm}$. The surface roughness measurements were performed with a cutoff length of 0.8 $\mathrm{mm}$. and an average of five readings was taken. The forms were removed using polynomial of the 3rd degree. The surface topography parameters were then calculated using software Talymap Gold. The parameters from ISO 25178 standard [14] were analyzed.

Height parameters:

- $\mathrm{Sq}$ - root mean square height of the surface,

- Ssk - skewness of height distribution,

- $\quad$ Sku - kurtosis of height distribution,

- Sp - maximum height of peaks,

- Sv - maximum height of valleys,

- $\mathrm{Sz}$ - maximum height of the surface,

- $\quad \mathrm{Sa}$ - arithmetical mean height of the surface

The surface texture parameters for the eleven experiment variants (and one after milling) are given in Tables 3. Figures 1,2 respectively show the chosen topography of the surface after burnishing for the smallest value of Sa parameter and threshold for burnishing and milling surface.

Table 3. The surface texture parameters for experiment variants and after milling.

\begin{tabular}{|c|c|c|c|c|c|c|c|c|c|c|c|c|c|}
\hline \multirow{2}{*}{$\begin{array}{c}\text { Expt. } \\
\text { No. }\end{array}$} & \multicolumn{3}{|c|}{ Coded } & \multicolumn{3}{|c|}{ Actual values } & \multicolumn{7}{|c|}{ Surface parameters } \\
\hline & $\mathrm{A}$ & $\mathrm{B}$ & $\mathrm{C}$ & $\begin{array}{c}\mathrm{P} \\
{[\mathrm{MPa}]}\end{array}$ & $\begin{array}{c}\mathrm{v} \\
{[\mathrm{mm} /} \\
\mathrm{min}]\end{array}$ & $\begin{array}{c}\mathrm{a} \\
{[\mathrm{mm}]}\end{array}$ & $\begin{array}{c}\mathrm{Sa} \\
{[\mu \mathrm{m}]}\end{array}$ & $\begin{array}{c}\mathrm{Sz} \\
{[\mu \mathrm{m}]}\end{array}$ & $\begin{array}{c}\mathrm{Sv} \\
{[\mu \mathrm{m}]}\end{array}$ & $\begin{array}{c}\mathrm{Sp} \\
{[\mu \mathrm{m}]}\end{array}$ & Sku & Ssk & $\begin{array}{c}\mathrm{Sq} \\
{[\mu \mathrm{m}]}\end{array}$ \\
\hline 1 & + & + & + & 20 & 3000 & 0.08 & 0.081 & 1.16 & 0.67 & 0.48 & 3.29 & 0.12 & 0.1 \\
\hline 2 & + & - & - & 20 & 1000 & 0.04 & 0.051 & 0.65 & 0.29 & 0.35 & 3.16 & 0.12 & 0.06 \\
\hline
\end{tabular}




\begin{tabular}{|c|c|c|c|c|c|c|c|c|c|c|c|c|c|}
\hline 3 & - & + & - & 10 & 3000 & 0.04 & 0.125 & 1.64 & 0.96 & 0.67 & 2.98 & -0.37 & 0.15 \\
\hline 4 & - & - & + & 10 & 1000 & 0.08 & 0.153 & 1.69 & 0.95 & 0.73 & 2.84 & -0.32 & 0.18 \\
\hline 5 & + & 0 & 0 & 20 & 2000 & 0.06 & 0.041 & 0.58 & 0.27 & 0.30 & 3.26 & 0.10 & 0.05 \\
\hline 6 & - & 0 & 0 & 10 & 2000 & 0.06 & 0.158 & 2.31 & 1.34 & 0.97 & 2.56 & -0.34 & 0.19 \\
\hline 7 & 0 & + & 0 & 15 & 3000 & 0.06 & 0.164 & 2.17 & 0.86 & 1.31 & 2.44 & -0.02 & 0.19 \\
\hline 8 & 0 & - & 0 & 15 & 1000 & 0.06 & 0.166 & 1.45 & 0.74 & 0.70 & 2.26 & 0.002 & 0.19 \\
\hline 9 & 0 & 0 & + & 15 & 2000 & 0.08 & 0.187 & 2.51 & 1.15 & 1.36 & 2.5 & 0.07 & 0.22 \\
\hline 10 & 0 & 0 & - & 15 & 2000 & 0.04 & 0.169 & 2.28 & 1.33 & 0.94 & 2.18 & -0.01 & 0.2 \\
\hline 11 & 0 & 0 & 0 & 15 & 2000 & 0.06 & 0.14 & 1.67 & 0.80 & 0.86 & 2.71 & 0.03 & 0.17 \\
\hline \multicolumn{7}{|c|}{ Surface after milling } & 0.6 & 5.5 & 2.7 & 2.8 & 3.52 & -0.19 & 0.73 \\
\hline
\end{tabular}
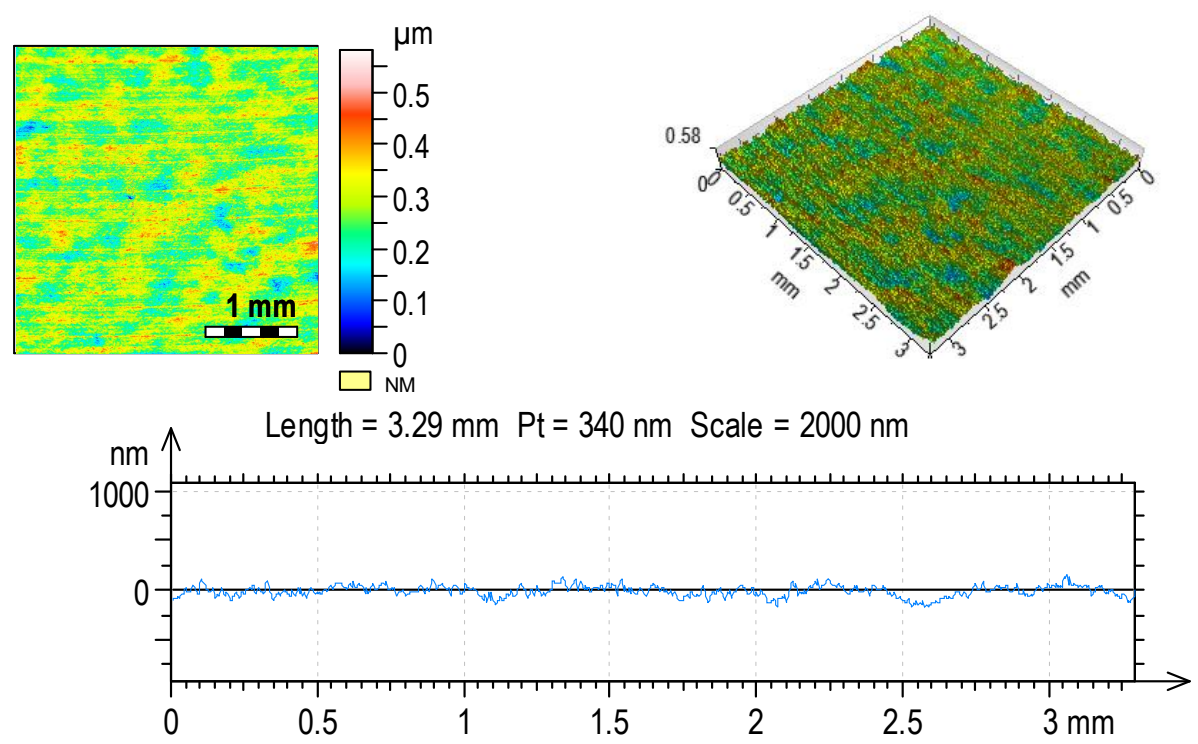

Fig. 1. Topography and surface profile of the surface after burnishing, expt. no. 5.
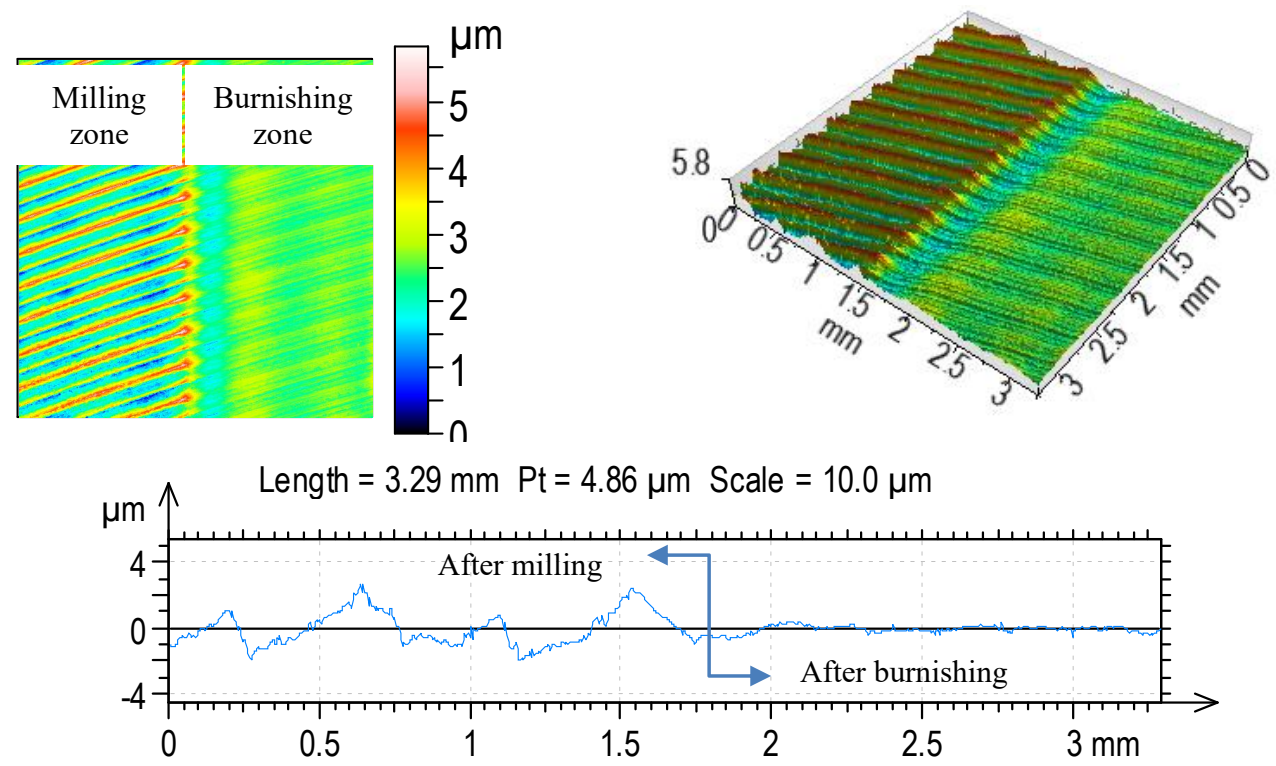

Fig. 2. Topography and surface profile of the threshold for burnishing and milling surface. 
It can be seen from the analysis of the surface topographies that a milling surface has an anisotropic characteristic. The ordinate distribution is similar to a Gaussian with low negative skewness $(\mathrm{Ssk}=-0.19$ and $\mathrm{Sku}=3.52)$. The following amplitude parameters were obtained on average: $\mathrm{Sa}=0.6 \mu \mathrm{m}, \mathrm{Sz}=5.5 \mu \mathrm{m}, \mathrm{Sp}=2.8 \mu \mathrm{m}$, and $\mathrm{Sv}=2.7 \mu \mathrm{m}$.

As a result of the ball burnishing, the height of the surface topography decreased. Depending on the machining conditions, the following amplitude parameters were obtained: $\mathrm{Sa}=0.041-1.87 \mu \mathrm{m}, \mathrm{Sz}=0.58-2.51 \mu \mathrm{m}, \mathrm{Sp}=0.306-1.36 \mu \mathrm{m}, \mathrm{Sv}=0.275-1.34 \mu \mathrm{m}, \mathrm{Sq}=$ $0.052-0.227 \mu \mathrm{m}$; however, the lowest values for the amplitude parameters $(\mathrm{Sq}=0.052 \mu \mathrm{m}$, $\mathrm{Sa}=0.041 \mu \mathrm{m}$, and $\mathrm{Sz}=0.052 \mu \mathrm{m}$ ) correspond to highest burnishing pressure $-20 \mathrm{MPa}$. For a burnishing pressure of $15 \mathrm{MPa}$, only one parameters reach the lowest values $\mathrm{Sku}=$ 2.18

Ssk parameteres represents the degree of symmetry of the surface heights about the mean plane. The sign of Ssk indicates the predominance of peaks (i.e. Ssk $>0$ ) or valley structures $(\mathrm{Ssk}<0)$ comprising the surface. Sku indicates the presence of inordinately high peaks/ deep valleys $(\mathrm{Sku}>3.00)$ or lack thereof $(\mathrm{Sku}<3.00)$ making up the texture.

After ball burnishing, the following parameter values describing the shape of the ordinate distribution were obtained: Ssk $=-0.376-0.123$, and $\mathrm{Sku}=2.18-3.29$. The Ssk parameter for burnishing pressures of $10 \mathrm{MPa}$ indicates a valley structure $(\mathrm{Ssk}<0)$, and for 15 and $20 \mathrm{MPa}$, a predominance of peaks (Ssk $>0$ ) occurs. For all experiments with burnishing pressure $20 \mathrm{MPa}$ the Sku parameters is above 3.0, which means height distribution is spiked.

The surface texture of the milled and burnished sections of the work piece is shown in figure 2. On the left side of the image, one can clearly observe the feed marks on the milled surfaces, area on the right side represents a smoother burnished surface. Comparing the value of $\mathrm{S}$ parameters for milled surface $(\mathrm{Sa}=0.6 \mu \mathrm{m})$ and the lowest value of $\mathrm{Sa}$ parameters after burnishing $(\mathrm{Sa}=0.041 \mu \mathrm{m})$ we can observe almost fifteen times smaller value.

As presented in table 3, the surface roughness appreciably decreases for high force value of $20 \mathrm{MPa}$ and further the surface roughness increases further for lower range 15-10 $\mathrm{MPa}$. This is due to the fact that at the commencement (when the force value is small), the burnishing ball penetrates a little distance into a work surface, causing a little or incomplete deformation of the asperities and thus resulting into reduced surface roughness.

The reduced surface roughness noticed at higher force ranges may be probably due to increased ball pressure on workpiece. Surface resulting in shrinking the most asperities and increasing the metal flow that leads to filling of more voids and/or valleys.

\section{Conclusions}

Ball burnishing with hydrostatic tools can be efficiently used to machine titanium alloy components Ti6Al4V with hardness of $36 \mathrm{HRC}$, provided proper selection of machining parameters. In this process, we can effectively modify the roughness of the surface. Proper selection of process parameters (significantly influenced by pressure, speed, width) allows reduce geometrical structure of the surface (height parameters).

The use of static determined, sequential, polyselective Hartley PS / DS-P: Ha3 plan enables clear identification of interdependencies between key process parameters and the properties of geometric structure of the surface. Ball burnishing process with hydrostatic tools leads to improvement of indicators of geometric surface structure. In most cases, the result is anisotropic surface, reduced roughness, reduced amplitude values of parameters such as: Sa, Sz. The biggest impact on the value of indicators of geometric structure comes from pressure, the smaller (but still significant) impact results from a tool speed and line to line pitch. 


\section{References}

1. Chunxiang C, BaoMin H, Lichen Z, Shuangjin L. Titaniumalloy production technology, market prospects and industrydevelopment. Mater Des (2011);32:1684-1691.

2. Pramanik A, Littlefair G. Machining of titanium alloy (Ti-6Al-4V) - theory to application. Machining Science and Technology Vol. 19, Iss. 1, (2015 )

3. Budinski KG. Tribological properties of titanium alloys. Wear (1991);151:203-17.

4. Yerramareddy S, Bahadur S. The effect of laser surfacetreatments on the tribological behavior of Ti-6Al-4V. Wear (1992);157:245-62.

5. Luca L.L., Neagu S.V., Marinescu I., Effects of working parameters on surface finish in ball-burnishing of hardened steels, Prec. Eng. 29 (2005) 253-256.

6. Avilés R., J. Albizuri, A. Rodríguez, L.N. López de Lacalle, Influence of low-plasticity ball burnishing on the high-cycle fatigue strength of medium carbon AISI 1045 steel, International Journal of Fatigue, Volume 55, (2013), 230-244,

7. Gharbi F., Sghaier S., Hamdi H., Ductility T. B., Improvement of aluminum 1050A rolled sheet by a newly designed ball burnishing tool device. Int J Adv Manuf Technol (2012) 60:87-99.

8. El-Tayeb N.S.M., Low K.O., Brevern P.V. Influence of roller burnishing contact width and burnishing orientation on surface quality and tribological behaviour of Aluminium 6061. Journal of Materials Processing Technology 186 (2007) 272-278.

9. Maawad E., Brokmeier H.-G., Wagner L., Sano Y., Genzel Ch. Investigation on the surface and near-surface characteristics of $\mathrm{Ti}-2.5 \mathrm{Cu}$ after various mechanical surface treatments. Surface \& Coatings Technology 205 (2011) 3644-3650.

10. Salahshoor M., Guo Y. B.. Process mechanics in ball burnishing biomedical magnesium-calcium alloy. Int J Adv Manuf Technol ., Volume 64, Issue 1-4, pp 133144

11. Malleswara Rao J.N., Chenna Kesava Reddy A., Rama Rao P. V., Experimental investigation of the influence of burnishing tool passes on surface roughness and hardness of brass specimens Indian Journal of Science and Technology Vol. 4 No. 9.

12. Avilés R., Albizuri J., Rodríguez A., López de Lacalle L.N., Influence of low-plasticity ball burnishing on the high-cycle fatigue strength of medium carbon AISI 1045 steel, International Journal of Fatigue, Volume 55, October 2013, 230-44,

13. Hartley H. O., Smallest composite design for quadratic response surface, Biometrics, $15 / 1959$.

14. ISO 25178: Geometric Product Specifications (GPS) - Surface texture: areal - Part 2: Terms, definitions and surface texture parameters. 